\title{
Endovascular embolization of arterial bleeding in patients with severe acute pancreatitis
}

Min Ai, GuangMing Lu, Jian Xu

Department of Medical Imaging, Jinling Hospital, Clinical School of Medical College, Nanjing University, Nanjing, Jiangsu Province, China

Videosurgery Miniinv 2019; 14 (3): 401-407

DOI: https://doi.org/10.5114/wiitm.2019.86919

\begin{abstract}
Introduction: Severe acute pancreatitis (SAP) has a high mortality rate of $20 \%$ to $30 \%$, with death often resulting from hemorrhage.

Aim: To investigate the role of digital subtraction angiography (DSA) and endovascular embolization in the management of arterial bleeding in SAP patients.

Material and methods: Seventy-six patients with SAP admitted to our hospital between January 2010 and May 2016 underwent DSA. DSA revealed arterial bleeding in 22 of these patients, who were treated with transcatheter endovascular embolization with coils and/or gelfoam particles. Patient demographics, angiographic features of vascular abnormalities, and outcomes of embolization were assessed.

Results: Arterial bleeding was the most common vascular abnormality (22/76 patients; 28.9\%). DSA enabled the identification of 27 bleeding arteries in 22 patients. The splenic artery was the most commonly affected vessel $(11 / 27 ; 40.7 \%)$. Among the 27 arteries treated with endovascular embolization, successful hemostasis was achieved in 96.3\% (26/27). Two patients developed major complications (hepatic and splenic abscess). These patients were treated with abdominal catheter drainage and anti-infection measures and ultimately recovered. The mean interval between initial onset of SAP and angiographic diagnosis of arterial bleeding was 56 days. Rebleeding was diagnosed in 5 patients (5/22; 22.7\%) during repeat angiography, with bleeding from new sites in four of these patients. The mean interval between successive angiography treatments was 38 days.

Conclusions: Endovascular embolization is a safe and effective method to localize bleeding arteries and achieve complete hemostasis in patients with SAP-related arterial bleeding.
\end{abstract}

Key words: hemorrhage, angiography, embolization, hemostasis, severe acute pancreatitis.

\section{Introduction}

Vascular complications, including hemorrhage, pseudoaneurysm formation, and venous thrombosis, are infrequent but serious sequelae of acute pancreatitis (AP) [1, 2]. Severe local inflammation and enzyme activation in patients with AP can result in arterial wall erosion, leading to acute arterial rupture or to pseudoaneurysm formation with subse- quent rupture. Arterial bleeding is the main cause of death in patients with AP, accounting for more than $50 \%$ of AP-related mortality [3].

Emergency intervention is necessary in patients with symptoms of arterial bleeding. Early surgical hemostasis has not been recommended because of high mortality caused by severe postoperative infection and high rebleeding rates resulting from iatrogenic arterial injury [4]. In these cases, urgent

\section{Address for correspondence}

Prof. Jian Xu, Department of Medical Imaging, Jinling Hospital, Clinical School of Medical College, Nanjing University, Nanjing 210002,

Jiangsu Province, China, e-mail: 13851656307@163.com 
endovascular embolization is generally considered the first-line treatment to manage bleeding, based on its high success rate and low mortality $[5,6]$.

Acute pancreatitis can be classified into three types: mild, moderately severe, and severe AP (SAP) [7]. Mortality and complication rates are highest among patients with SAP, which is characterized by persistent organ failure affecting one or more organ

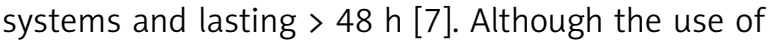
embolization to control arterial bleeding in patients with AP has been well evaluated, information on treatment in patients with SAP is scarce. Given the specificity and severity of SAP, the clinical manifestations of arterial bleeding in patients with SAP may differ from those in patients with other types of AP. If confirmed, these differences would be important clinical considerations in treatment.

\section{Aim}

The aim of this study is to investigate the role of digital subtraction angiography (DSA) and endovascular embolization in the management of arterial bleeding in SAP patients.

\section{Material and methods}

Seventy-six patients were admitted to our hospital with SAP between January 2010 and May 2016 and underwent emergency DSA to screen for arterial bleeding. DSA revealed arterial bleeding in 22 of these patients, who were then treated with endovascular embolization. Because this was a retrospective study, ethical approval from our institution was not required. Informed consent for DSA was obtained from each patient and recorded in the electronic medical record during hospitalization. Data on patient demographics, angiographic features of arterial abnormalities, and outcomes of embolization were collected from the medical records.

Percutaneous puncture of the right common femoral artery was performed by an interventional physician. After placement of a 5-French (Fr) sheath (Terumo Corporation, Japan) in the right common femoral artery, the celiac trunk, superior mesenteric artery (SMA), and inferior mesenteric artery (IMA) were catheterized with a $5-\mathrm{Fr}$ angiographic catheter (RH catheter; Cook, USA) for angiographic localization of the bleeding artery.

If angiography showed contrast agent leakage from an artery, we immediately inserted a $2.6-\mathrm{Fr}$ microcatheter (Stride microcatheter; Asahi Corporation, Japan) into a branch vessel of the affected artery, and then repeated angiography to evaluate possible rupture of the affected artery. When angiography clearly demonstrated arterial rupture and bleeding, an appropriate volume of gelfoam particles (Xiangen Medical Technology, China) was immediately injected through the 2.6- $\mathrm{Fr}$ microcatheter to embolize the affected vessel until the contrast agent showed "return to flow", at which time embolization was stopped. If complete embolization was difficult to achieve with gelfoam particles, a microcoil (Cook, USA) was used for embolization.

\section{Results}

The average age of the 76 patients was 46 years. Most patients were male (54/76; 71.05\%). Biliary pancreatitis was the main type of AP (39/76; 51.3\%); other etiologies included hyperlipemia (25/76; $32.9 \%)$, alcohol intake ( $7 / 76 ; 9.2 \%)$, pregnancy $(1 / 76$; $1.3 \%)$, trauma ( $1 / 76 ; 1.3 \%)$, and undetermined ( $3 / 76$; $3.9 \%)$. At the time of SAP diagnosis, the average serum amylase was $257 \mathrm{U} / \mathrm{l}$ and serum lipase was $875 \mathrm{U} / \mathrm{l}$. The most common presenting complaint was acute abdominal pain (32/76; 42.1\%). Patients' demographic and clinical data are listed in Table I.

The angiographic findings are listed in Table II. Vascular complications were categorized into three types: intra-abdominal visceral arterial hemorrhage, pseudoaneurysm, and venous thrombosis. In our patients, the distribution of these complications was $28.9 \%$ (22/76), $14.5 \%$ (11/76), and 25.0\% (19/76), respectively.

Among the 22 patients with arterial hemorrhage, there were 27 bleeding arteries, predominantly the splenic artery $(11 / 27 ; 40.7 \%)$, followed by the SMA $(4 / 27 ; 14.8 \%)$, the gastroduodenal artery (4/27; $14.8 \%)$, the IMA $(3 / 27 ; 11.1 \%)$, the pancreaticoduodenal artery $(2 / 27 ; 7.4 \%)$, the left hepatic artery $(1 / 27 ; 3.7 \%)$, the right hepatic artery $(1 / 27 ; 3.7 \%)$, and the right inferior phrenic artery $(1 / 27 ; 3.7 \%)$.

Table III shows the results of embolization procedures. A total of 27 endovascular embolization procedures were performed in 22 patients; 5 patients underwent two rounds of treatment. Endovascular embolization was performed with coils and/or gelfoam particles in all patients (Photo 1). The embolization success rate was $96.3 \%$ (26/27); one embolization failed. One patient (1/22; 4.5\%) developed 
Table I. Demographics, clinical findings, and laboratory findings of 76 patients with severe acute pancreatitis who underwent digital subtraction angiography

\begin{tabular}{|c|c|}
\hline Parameter & Value \\
\hline Number of patients & 76 \\
\hline Age, mean (range) [years] & $46(20-67)$ \\
\hline Male : female ratio & $54: 22$ \\
\hline \multicolumn{2}{|l|}{ Causes of SAP: } \\
\hline Biliary & $51.3 \%(39 / 76)$ \\
\hline Hyperlipidemic & $30.3 \%(23 / 76)$ \\
\hline Alcoholic & $6.6 \%(5 / 76)$ \\
\hline Traumatic & $1.3 \%(1 / 76)$ \\
\hline Pregnancy-induced & $1.3 \%(1 / 76)$ \\
\hline Unknown & $9.2 \%(7 / 76)$ \\
\hline \multicolumn{2}{|c|}{ Clinical presentation at admission: } \\
\hline Acute abdominal pain & $42.1 \%(32 / 76)$ \\
\hline Fever & $34.2 \%(26 / 76)$ \\
\hline Bleeding from drains & $10.5 \%(8 / 76)$ \\
\hline Shock & $5.3 \%(4 / 76)$ \\
\hline Melena & $5.3 \%(4 / 76)$ \\
\hline Hematemesis & $1.3 \%(1 / 76)$ \\
\hline Intestinal fistula & $1.3 \%(1 / 76)$ \\
\hline \multicolumn{2}{|c|}{ Laboratory abnormalities, mean [U/I]: } \\
\hline Amylase before intervention & $252(30-1880)$ \\
\hline Lipase before intervention & $882(15-11312)$ \\
\hline Hospital stay, mean [days] & $38(2-205)$ \\
\hline
\end{tabular}

a hepatic abscess and one (1/22; 4.5\%) developed a splenic abscess (Photo 2). These patients were treated with prolonged drainage and anti-infection measures, and ultimately recovered. Two patients $(2 / 22,9.1 \%)$ developed splenic infarction and recovered with conservative treatment.

Arterial bleeding occurred in the 22 patients after a mean of 56 days from the initial onset of SAP. Five patients $(5 / 22 ; 22.7 \%)$ experienced recurrent bleeding, which occurred on days $30,20,10,13$, and 118 , respectively, after the first angiography. The mean interval until rebleeding was 38 days. Among rebleeding patients, four patients develop arterial
Table II. Angiographic findings

\begin{tabular}{|lc|}
\hline Parameter & Results \\
\hline Angiographic finding: & $28.9 \%(22 / 76)$ \\
\hline Arterial bleeding & $14.5 \%(11 / 76)$ \\
\hline Pseudoaneurysm formation & $25.0 \%(19 / 76)$ \\
\hline Venous thrombosis & 27 \\
\hline Number of bleeding arteries & $40.7 \%(11 / 27)$ \\
\hline Angiographic location: & $14.8 \%(4 / 27)$ \\
\hline Splenic artery & $14.8 \%(4 / 27)$ \\
\hline Sustroduodenal artery & $11.1 \%(3 / 27)$ \\
\hline Inferior mesenteric artery & $7.4 \%(2 / 27)$ \\
\hline Lancreaticoduodenal artery & $3.7 \%(1 / 27)$ \\
\hline Right hepatic artery & $3.7 \%(1 / 27)$ \\
\hline Right inferior phrenic artery & $3.7 \%(1 / 27)$ \\
\hline
\end{tabular}

Table III. Outcomes of angiographic embolization

\begin{tabular}{|lc|}
\hline Parameter & Results \\
\hline Case of hemorrhage: & 22 \\
\hline Interval, mean [days] & 56 \\
\hline Rebleeding cases: & $22.7 \%(5 / 22)$ \\
\hline Interval, mean [days] & 38 \\
\hline \begin{tabular}{l} 
Number of embolizations: \\
\hline Success rate
\end{tabular} & 27 \\
\hline \begin{tabular}{l} 
Complications: \\
\hline Splenic infarction
\end{tabular} & $96.3 \%(26 / 27)$ \\
\hline \begin{tabular}{l} 
Splenic abscess \\
\hline Hepatic abscess
\end{tabular} & $4.5 \%(1 / 22)$ \\
\hline
\end{tabular}

Mean interval - Interval between initial onset of SAP and angiographic diagnosis of bleeding; interval between successive angiographies in five patients with rebleeding.

bleeding from a site different from that at the initial angiogram.

\section{Discussion}

There are two phases in the course of SAP: early and late. The early phase usually lasts for the first 

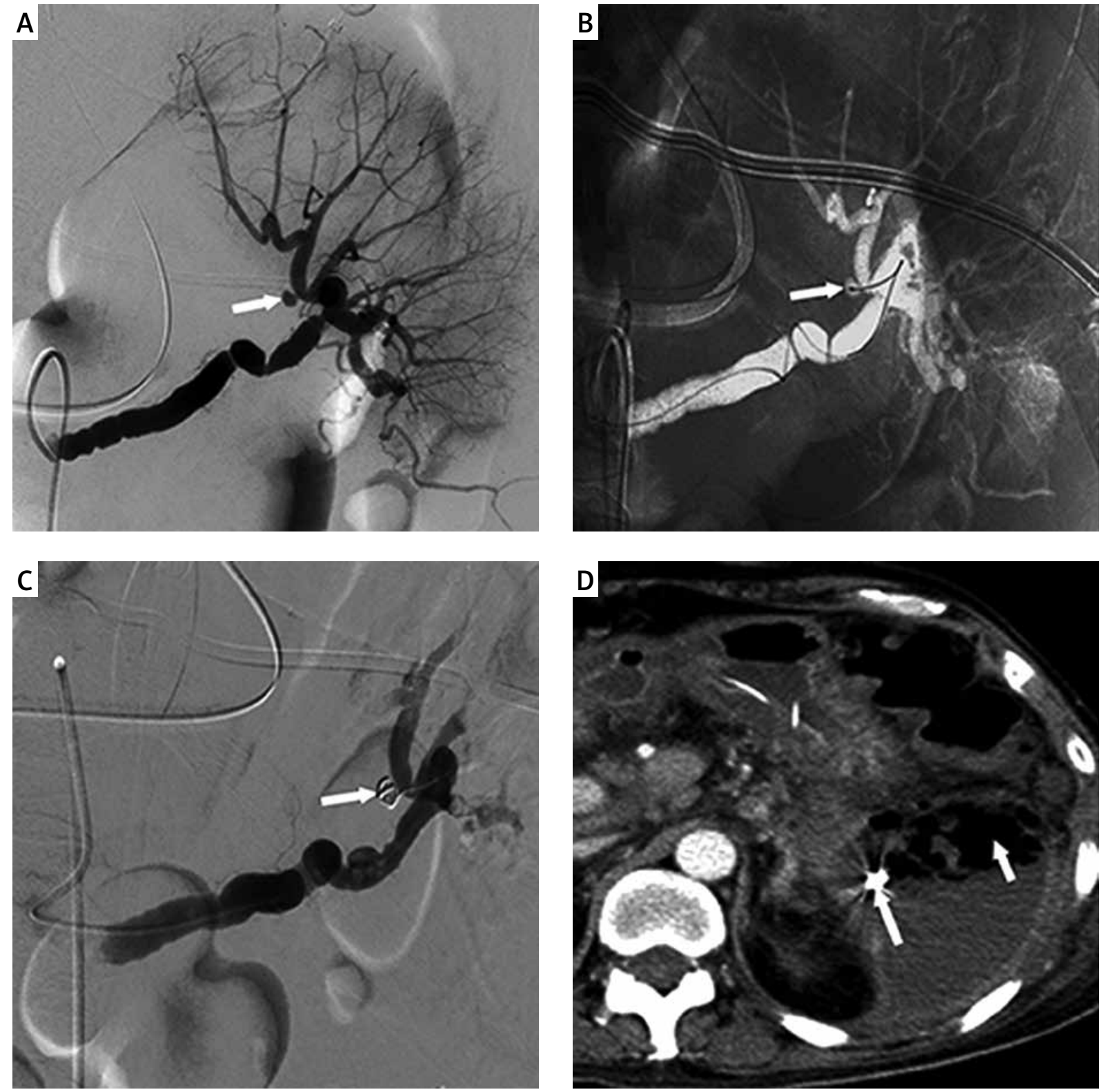

Photo 1. Pseudoaneurysms of the splenic artery. A - Angiogram of splenic artery. Contrast within the aneurysm sac (white arrow). B - Microcatheter was placed in the aneurysm sac (white arrow). C - Angiogram after embolization. Coils were placed in the aneurysm sac (white arrow) - successful aneurysm exclusion without aneurysm refilling. D - CT image obtained during follow-up examination 1 week after the embolization. The embolization coil position is indicated (long white arrow). This patient developed a splenic abscess. The abscess cavity contains gas (short white arrow)

week after admission and the late phase can extend for weeks or months. SAP patients often develop local complications during the late phase, including acute peripancreatic fluid collection, pancreatic pseudocyst, acute necrotic collection, and walled-off necrosis [8]. Corrosive infectious fluids contained in these local lesions can damage arterial walls and raise the arterial bleeding risk in patients; therefore, long-term monitoring for local complications is necessary [3, 9, 10]. Local complications can be confirmed by their characteristic manifestations on contrast-enhanced computed tomography (CECT) [7]. In our group of patients, CECT was performed repeatedly during hospitalization to assess SAP disease progression. 

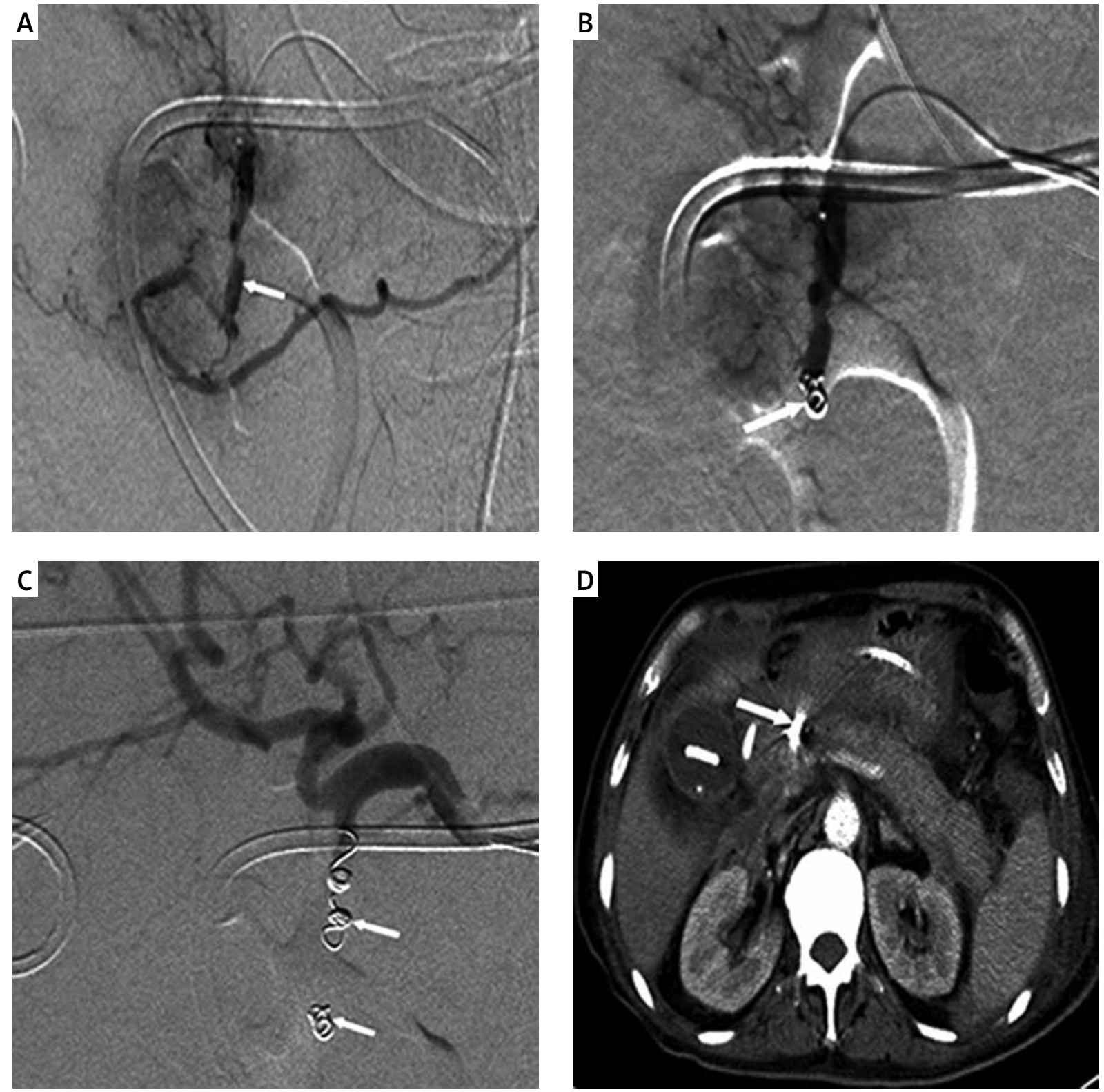

Photo 2. Pseudoaneurysms of the gastroduodenal artery. A - Angiogram of gastroduodenal artery. Contrast within the aneurysm sac (white arrow). B - Coils were placed in the distal vessel of aneurysm sac (white arrow). C - Angiogram after embolization. Other coils were placed in the proximal vessel of aneurysm sac (white arrow) - successful aneurysm exclusion. D - CT image obtained during follow-up examination 1 month after the embolization. The embolization coil position is indicated (white arrow)

When patients with SAP showed clinical signs of potentially fatal arterial bleeding, such as drainage-tube bleeding, hematemesis, or a sharp decline in hemoglobin, we performed emergency DSA. The bleeding rate in AP ranges from $1.2 \%$ to $14.5 \%$ [2]; however, data on bleeding rates for SAP are lacking. The frequency of bleeding among patients admitted with SAP was $28.9 \%$ in the present study, which is markedly higher than the rate among patients with other forms of AP. This difference may be attributed to two factors. First, corrosive infectious fluids in later-phase SAP make patients more vulnerable to infection and sepsis, which can cause hyperfibrinolysis and subsequent hemorrhage [11]. Second, the high rate of hemorrhagic complications in our patients may have resulted from associated coag- 
ulation disorders. Blood loss during surgery results in the intravascular consumption of clotting factors, the concentrations of which are further reduced by the large infusion volumes needed to improve hemodynamic stability. This, in turn, increases the risk of hemorrhage.

What is the optimal treatment protocol to resolve arterial bleeding in patients with SAP? It is widely accepted that early surgical intervention markedly increases both mortality and rebleeding rates [12-15]. Endovascular embolization is generally considered the preferred approach to managing hemorrhage in AP patients [5, 16, 17]. Kim et al. [6] reported the following distribution of affected arteries in patients with pancreatitis: the splenic artery in $34.8 \%$ of cases, the gastric artery in $17.4 \%$, the pancreaticoduodenal artery in $17.3 \%$, the gastroduodenal artery in $15.2 \%$, and others (SMA, jejunal, colic, inferior phrenic, and hepatic arteries) in $2.2 \%$ to $4.3 \%$. Splenic artery involvement was also predominant in our study (40.7\%); however, the rates of SMA (14.8\%) and IMA (11.1\%) involvement were higher and that of the pancreaticoduodenal artery (7.4\%) was lower than previously reported [6]. Our results suggest that without early angiographic localization, a related affected artery may be missed at surgery. Hepatic and phrenic artery involvement, which occurred in our patients, is rarely reported in SAP but may be related to the diffusion of inflammatory mediators and activated pancreatic enzymes.

Arterial bleeding usually occurs in the later phase of SAP (> 7 days) $[18,19]$. In our patients, angiographic diagnosis of arterial bleeding occurred at a mean of 56 days after the initial onset of SAP. This time point may be a useful guide for clinicians to evaluate the possibility of potentially fatal arterial bleeding and treat it accordingly.

The reported success rate of angiographic embolization ranges from $79 \%$ to $100 \%[20,21]$. In our series, the technical success rate was $96.3 \%$. As pancreatic enzymes corrode the arterial wall, peripancreatic arteries become susceptible to external stimulation in patients with SAP. Embolization failed in one patient because the affected vessel became spastic in response to repeated microcatheter insertion, which illustrates the need for gentle interventional operations. Two patients died of hemorrhagic shock and one of brain stem infarction. All three deaths reflect coagulation system dysfunction in
SAP. None of the deaths was related to the embolization procedure.

Complications after embolization are infrequent [6]. Splenic infarction is of little clinical significance and typically resolves spontaneously or with conservative treatment [22]. However, visceral abscesses are a serious complication of embolization. One (4.5\%) patient in our study developed a hepatic abscess with embolization of the left hepatic artery. This patient and another with splenic abscess were treated with prolonged drainage and anti-infection measures, and ultimately recovered.

Rebleeding after embolization increases the difficulty of treating patients with SAP. Reported rebleeding rates range from $0 \%$ to $11 \%$ [23-26]; the rebleeding rate in the present study was $22.7 \%$, which is markedly higher than reported rates. Local infected fluid collection is the most common risk factor for arterial bleeding and is more common in SAP than in other types of $\operatorname{AP}[3,9,10]$, which explains why rebleeding is more common among patients with SAP. Minimally invasive drainage of peripancreatic infectious liquid has been an effective means of preventing bleeding recurrence after embolization $[27,28]$. Necrosectomy may also be performed when necessary. However, because postoperative mortality is high after necrosectomy, the procedure should be postponed until 1 month after hospital admission, when a clear boundary has formed between infectious liquid and normal tissue. At that point, the risk of inflammatory diffusion and postoperative sepsis is markedly lower than at earlier disease stages [29]. In our study, five recurrences occurred within 4 months (mean: 38 days) after initial embolization, indicating that this critical time period may be important in patient follow-up. However, more data are needed to confirm this finding.

This study had three main limitations. First, this was a nonrandomized retrospective study; its retrospective nature may have resulted in a lower reported incidence of hemorrhage than the actual incidence. Second, the study included only patients who underwent embolization with coils and/or gelfoam particles. A controlled study that includes other types of embolic agents is needed. Finally, the relatively small number of patients in this study may limit the generalizability of our findings; more cases are needed to confirm our findings. Nevertheless, to our knowledge, this is the largest reported series of patients with SAP to date. 


\section{Conclusions}

Arterial bleeding occurs more frequently in patients with SAP than in those with other types of AP. Arterial bleeding can be a fatal complication; emergency intervention should be performed to avoid poor outcomes. Endovascular embolization is safe and efficient for controlling potentially fatal arterial bleeding, and can be considered first-line treatment in the management of arterial bleeding in patients with SAP.

\section{Conflict of interest}

The authors declare no conflict of interest.

\section{References}

1. Czernik M, Stefańczyk L, Szubert W, et al. Endovascular treatment of pseudoaneurysms in pancreatitis. Videosurgery Miniinv 2014; 13: 138-44.

2. Mallick IH, Winslet MC. Vascular complications of pancreatitis. JOP 2004; 5: 328-37.

3. Flati G, Andrén-Sandberg Å, La Pinta M, et al. Potentially fatal bleeding in acute pancreatitis: pathophysiology, prevention, and treatment. Pancreas 2003; 26: 8-14.

4. Kriwanek S, Gschwantler M, Beckerhim P, et al. Complications after surgery for necrotizing pancreatitis: risk factors and prognosis. Eur J Surg 1999; 165: 952-7.

5. Barge JU, Lopera J E. Vascular complications of pancreatitis: role of interventional therapy. Korean J Radiol 2012; 13 Suppl 1: S45.

6. Kim J, Shin JH, Yoon HK, et al. Endovascular intervention for management of pancreatitis-related bleeding: a retrospective analysis of thirty-seven patients at a single institution. Diagn Interv Radiol 2015; 21: 140-7.

7. Banks PA, Bollen TL, Dervenis C, et al. Classification of acute pancreatitis - 2012: revision of the Atlanta classification and definitions by international consensus. Gut 2013; 62: 102-11.

8. Wysocki L, Wroński M, Cebulski W, et al. Combined minimally invasive management of infected pancreatic necrosis: a case report. Videosurgery Mininv 2014; 9: 107-9.

9. Gloor B, Müller CA, Worni M, et al. Late mortality in patients with severe acute pancreatitis. Br J Surg 2001; 88: 975-9.

10. Bello B, Matthews JB. Minimally invasive treatment of pancreatic necrosis. World J Gastroenterol 2012; 18: 6829-35.

11. Fourrier F. Severe sepsis, coagulation, and fibrinolysis: dead end or one way? Crit Care Med 2012; 40: 2704-8.

12. Wei AL, Guo Q, Wang MJ, et al. Early complications after interventions in patients with acute pancreatitis. World J Gastroenterol 2016; 22: 2828-36.

13. Beattie GC, Mason J, Swan D, et al. Outcome of necrosectomy in acute pancreatitis: the case for continued vigilance. Scand J Gastroenterol 2002; 37: 1449-53.

14. Mier J, Luque-de León E, Castillo A, et al. Early versus late necrosectomy in severe necrotizing pancreatitis. Am J Surg 1997; 173: 71-5.
15. Hartwig W, Maksan SM, Foitzik T, et al. Reduction in mortality with delayed surgical therapy of severe pancreatitis. J Gastrointest Surg 2002; 6: 481-7.

16. Barge JU, Lopera JE. Vascular complications of pancreatitis: role of interventional therapy. Korean J Radiol 2012; 13: S45-55.

17. Phillip V, Rasch S, Gaa J, et al. Spontaneous bleeding in pancreatitis treated by transcatheter arterial coil embolization: a retrospective study. PLoS One 2013; 8: e72903.

18. Andersson E, Ansari D, Andersson R. Major haemorrhagic complications of acute pancreatitis. Br J Surg 2010; 97: 1379-84.

19. Chen Y, Zhou J, Li G, et al. Early spontaneous abdominal bleeding is associated with poor outcome in moderate to severe acute pancreatitis patients: a propensity matched study. Sci Rep 2017; 7: 42607.

20. Hsu JT, Yeh CN, Hung CF, et al. Management and outcome of bleeding pseudoaneurysm associated with chronic pancreatitis. BMC Gastroenterol 2006; 6: 3.

21. Zyromski NJ, Vieira C, Stecker M, et al. Improved outcomes in postoperative and pancreatitis-related visceral pseudoaneurysms. J Gastrointest Surg 2007; 11: 50-5.

22. Kirby JM, Vora P, Midia M, et al. Vascular complications of pancreatitis: imaging and intervention. Cardiovasc Interv Radiol 2008; 31: 957-70.

23. Sethi H, Peddu P, Prachalias A, et al. Selective embolization for bleeding visceral artery pseudoaneurysms in patients with pancreatitis. Hepatobiliary Pancreat Dis Int 2010; 9: 634-8.

24. Bergert H, Dobrowolski F, Caffier S, et al. Prevalence and treatment of bleeding complications in chronic pancreatitis. Langenbecks Arch Surg 2004; 389: 504-10.

25. Nicholson AA, Patel J, McPherson S, et al. Endovascular treatment of visceral aneurysms associated with pancreatitis and a suggested classification with therapeutic implications. J Vasc Interv Radiol 2006; 17: 1279-85.

26. Deshmukh H, Rathod K, Garg A, et al. Transcatheter embolization as primary treatment for visceral pseudoaneurysms in pancreatitis: clinical outcome and imaging follow up. Indian J Gastroenterol 2004; 23: 56-8.

27. Smoczyński M, Jagielski M, Jabłońska A, et al. Transpapillary drainage of walled-off pancreatic necrosis - a single center experience. Videosurgery Miniinv 2016; 11: 527-33.

28. Szeliga J, Jackowski M. Minimally invasive procedures in severe acute pancreatitis treatment - assessment of benefits and possibilities of use. Videosurgery Miniinv 2014; 9: 170-8.

29. Pezzilli R, Zerbi A, Campra D, et al. Consensus guidelines on severe acute pancreatitis. Dig Liver Dis 2015; 47: 532-43.

Received: 23.04.2019, accepted: 4.06.2019. 